\title{
A Model for General Collective Intelligence
}

Andy E. Williams

Nobeah Foundation, Nairobi, Kenya

Email: awilliams@nobeahfoundation.org

\begin{abstract}
A General Collective Intelligence or GCI is a hypothetical platform that combines groups into a virtual collective cognition with a single well-defined thread of collective reasoning. While groups might have some innate general problem-solving ability described by a general collective intelligence factor (c), any collective intelligence software platform or methodology used as a decision-making tool by groups to increase problem-solving ability has narrow problem-solving ability where it is not able to address any problem in general. As opposed to such collective intelligence methodologies or software platforms, GCI has the potential to combine individual cognition into a single virtual collective cognition with general problem-solving ability, and also creates the potential to exponentially increase this general problem-solving ability.
\end{abstract}

\section{Introduction}

Research suggests that groups of individuals have an innate degree of general problem ability that has been described by some collective intelligence researchers as the "general intelligence factor (c)" characteristic of each group [1]. Similar to the $\mathrm{g}$ factor (g) for general individual intelligence, these collective intelligence researchers have attempted to extract this general collective intelligence factor (c factor) for groups as an indication of a group's ability to perform a wide range of tasks. This $\mathrm{c}$ factor has been interpreted as the intelligence quotient for groups (Group-IQ).

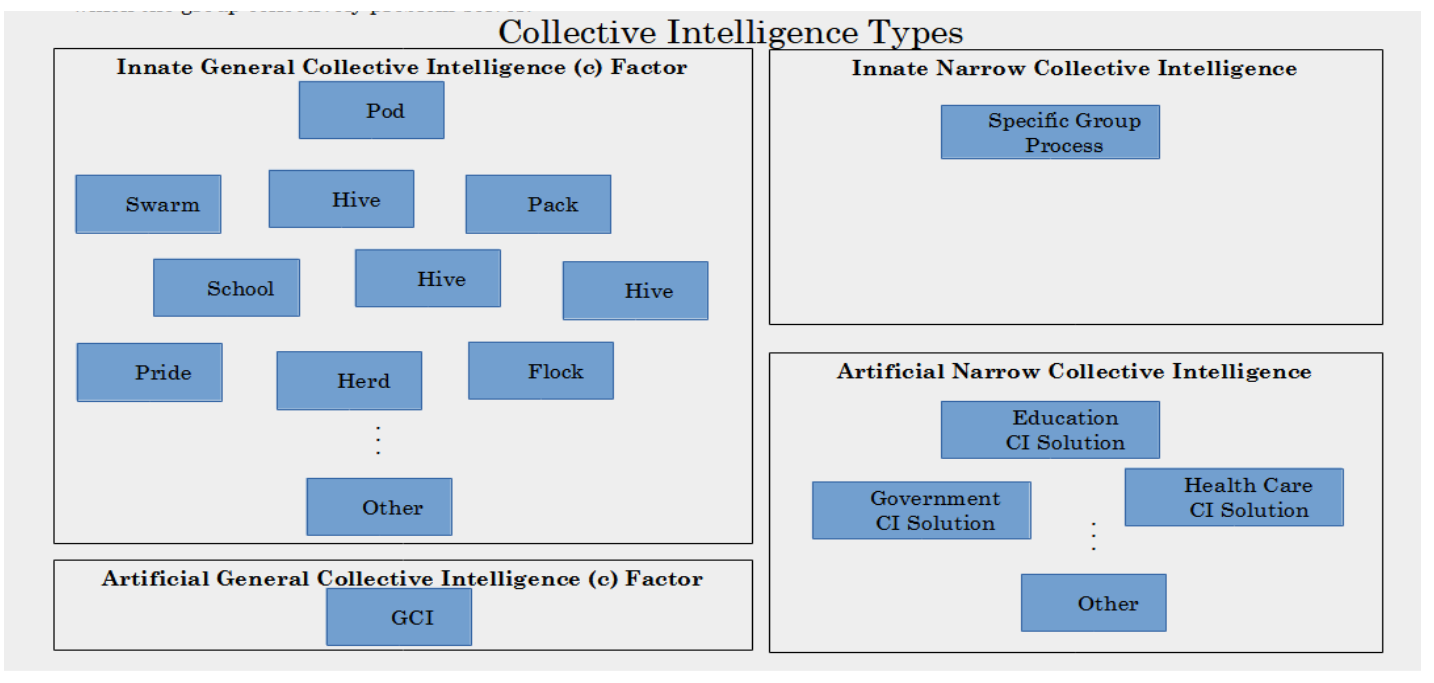

Figure 1: Types of collective intelligence.

A model of the mechanisms by which an innate general collective intelligence factor might arise has been proposed [2]. In addition to the innate general collective factor of each group, collective intelligence platforms potentially provide groups with increased problem-solving ability in some narrower range of problems, such as medical diagnosis [3], or management of agricultural resources [4]. However, since the introduction of the concept of a "collective superintelligence"[5], a number of methodologies by which that general collective intelligence factor might be increased into a "collective 
superintelligence" have also been proposed. Thomas Malone of the MIT Center for Collective Intelligence (CCI) has associated the concept of an extremely high group general intelligence factor with that of a supermind [6]. Others, have proposed the concept of a "global brain" [7][8]. More recently the concept of a General Collective Intelligence or GCI has been defined as a model of collaborative computing that might be implemented by a software platform to significantly increase the general problem-solving ability of the group so that it is significantly greater than that of any individual. The model of GCI is potentially a radical departure from the model of innate general collective intelligence, and from the "collective superintelligence" and "global brain theory" methodologies that have been suggested to have the potential to greatly increase this general collective intelligence. The difference being that the GCI model quantifies intelligence, and defines a specific set of functionality by which superintelligence might be achieved, to exponentially increase the general problem-solving ability of groups as compared to any individual.

\section{Deriving the Model of General Collective Intelligence}

Any dynamical system with repeatable behavior can potentially be modeled in a human-centric way in terms of the minimal set of functions that can be used to represent all the human observable behavior of the system. All the states that can be accessed by the system through such functions then form a "functional state space" which the system moves through through as in figure 2.

\section{Path Through Functional State Space}

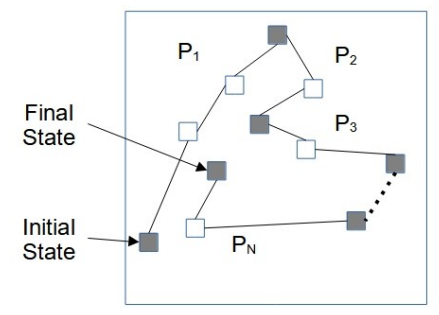

Figure 2: In Human-Centric Functional Modeling systems are represented as moving through a functional state space.

Any system with a stable set of repeatable functions also must stay within a bounded region of a "fitness space" that describes the fitness of the system to execute its functions. Defining a "fitness space" in terms of target value of fitness, actual value of fitness, and predicted value of fitness of the system that is achieved as a result of some action (some path) in functional state space, the path through this fitness space must stay within a bounded region in fitness space. In this sense the motion in fitness space must be stable globally throughout the fitness space, despite potentially being chaotic in functional state space due to random interactions with the environment as in figure 3 . 


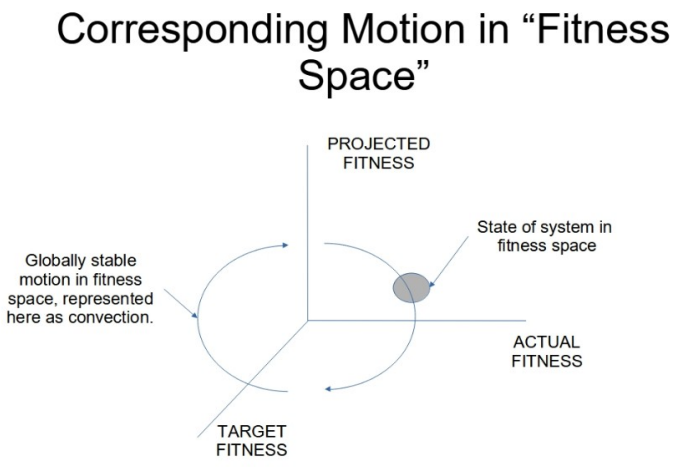

Figure 3: In Human-Centric Functional Modeling systems are represented as also moving through a fitness space as they move through functional state space.

All the processes of life, beginning with homeostasis, and ending in consciousness, and cognition, can potentially be modeled in this same way as having dynamics that are confined to a bounded region in these three dimensions. Representing each process as an adaptive process that operates within a particular adaptive domain, and representing each process as being implemented by a functional component, life is then a hierarchy of adaptive processes implemented by a hierarchy of functional components.

From this functional modeling perspective the cognitive system moves through a space of concepts or a "conceptual space". A collective cognition can then be defined as a process moving through the collective conceptual space. Because this "Human-Centric Functional Modeling (HCFM)" approach is itself independent of implementation, it has been used not only to define a model of individual human cognition [9],[10] as well as a model of Artificial General Intelligence (AGI) [11], but also the model of collective cognition described in this paper, which is intended to potentially be replicated in software platforms that organize individuals into a Collective intelligence $(\mathrm{CI})$ with general problem solving ability (a General Collective Intelligence or GCI). All of these models (AGI, CI, GCI, human cognition) are defined in terms of their dynamics through the functional state space and fitness space of the individual or collective cognitive system.

The functions of individual cognition in this model consist of a number of functional units (F1 to F3) that process neural signals into concepts, and in doing so map to and from signal space to the conceptual space. The functions also consist of a number of functional units that process concepts (F4 to F7 and FS consisting of storage (memory), recollection, recognition, etc.,) and that can be used to compose any reasoning process. In doing so they receive concepts from the functional state space of the cognitive system ("conceptual space") as input, and produce other concepts as output to that "conceptual space". By executing reasoning processes defined in terms of these functions the cognitive system navigates this conceptual space. Being a functional model independent of implementation, this model is also proposed to be applicable to a system of collective cognition. A system of collective cognition is then defined as navigating the collective conceptual space with these same functions as well as navigating the collective fitness space.

Modeling conceptual space in this way as a graph containing a network of nodes in which each node represents a concept and in which the edges connecting nodes represent the reasoning processes which define the meaning of those concepts, is useful because such a graph potentially defines the first completely self-contained representation of the meaning of those concepts and reasoning processes. Or in other words, that graph potentially defines the first complete semantic model. If any individual 
reasoning process can be represented as a path through an individual's conceptual space, then since collective reasoning consists of individual reasoning, all collective reasoning can be modeled as a path of the collective cognition through the collective conceptual space. Modeling collective reasoning processes in terms of complete semantic models makes it potentially possible to exchange meaning rather than just information, so that meaning might be exchanged at a far greater rate and scale.

This capacity to represent the state of any system of collective cognition as a point in a collective conceptual space, and this capacity to represent any collective reasoning process as a path through that collective conceptual space, enables collective intelligence solutions to be unambiguously modeled, so they can potentially be added to a library that a system of general collective intelligence can use to increase its general problem solving ability. In addition, breaking systems down to a set of discrete, objectively defined functions that can be independently implemented by people from different disciplines carries the benefits common to functional modeling approaches [12] - [18], namely that it may facilitate massive interdisciplinary cooperation to achieve this implementation, where such cooperation may not yet have proven possible otherwise.

In an individual cognition, the complete set of cognitive functions is represented as beginning on the input processing path (processing cognition of sensory or other input) with cognitive functions that are act to receive understanding in terms of concepts (understanding meaning the process that enables comprehension of the sentence "the quick brown fox jumped over the lazy dog"). On the output path the inverse of these cognitive functions are represented as directing reasoning (reasoning meaning the process that enables answering the question "what fox jumped over what dog?").

\begin{tabular}{|c|c|c|}
\hline \multicolumn{3}{|c|}{ Functional Units in Systems of Cognition } \\
\hline Functional Unit & Input Function & Output Function \\
\hline $\mathrm{F} 1$ to $\mathrm{F} 3$ & Create Concept & Create Signals from Concept \\
\hline F4 & STORE (Store Concept) & $\begin{array}{l}\text { DECOMPOSE STORAGE } \\
\text { (Determine Concept in Storage } \\
\text { Function) }\end{array}$ \\
\hline F5 & RECALL (Recall Concept) & $\begin{array}{l}\text { DECOMPOSE RECALL } \\
\text { (Determine Concept in Recall } \\
\text { Function) }\end{array}$ \\
\hline F6 & $\begin{array}{l}\text { DETECT PATTERN (Detect } \\
\text { Pattern in Concept) }\end{array}$ & $\begin{array}{l}\text { DECOMPOSE PATTERN (Detect } \\
\text { Concept in Pattern) }\end{array}$ \\
\hline F7 & $\begin{array}{l}\text { DETECT SEQUENCE (Detect } \\
\text { Sequence of Patterns in Concept) }\end{array}$ & $\begin{array}{l}\text { DECOMPOSE SEQUENCE (Detect } \\
\text { Concept in Sequence of Patterns) }\end{array}$ \\
\hline FS & $\begin{array}{l}\text { COGNITIVE AWARENESS (Ch } \\
\text { Reasoning Process to Solve it) }\end{array}$ & loose Problem to Solve and \\
\hline
\end{tabular}

Table 1: Functional units in systems of individual or collective cognition proposed by the Functional Modeling Framework (FMF).

Human reasoning consists of type 1 (intuitive) reasoning and type 2 (rational methodical) reasoning [19]. In order for the above set of reasoning functions to be considered complete it must have the capacity to represent any possible reasoning process. Representation of any reasoning processes might be possible because any intuitive reasoning can be represented in a functional model as a form of pattern detection, which can be represented by F6. And since the set of functions AND, OR, as well as NOT can represent all logic and is therefore a Turing complete set of operations, any logic, and therefore the logic in any rational methodical reasoning process, might be represented in a functional model in terms of a function to detect patterns as concepts, and in terms of patterns representing a 
Turing complete set of logic operations on concepts, whether or not they are the functions AND, OR, and NOT.

Functions of the Collective Cognition

In order to navigate the collective conceptual space with general problem-solving ability a requirements analysis [20] suggests the collective cognition must have the functional elements described in table 2:

\section{Component of GCI Model Description}

Functional modeling Components are modeled only by function to remove prejudice for or

(problems and solutions) against any given implementation.

Functional decomposition Functional components are decomposed into their most basic functional building blocks for reuse.

Functional domain bridging Different domains in which different functions are more fit in achieving the same purpose are identified. These domains are bridged by using a set of weights which identify the best function in each domain.

Functional fitness Every functional component is assigned some projected and actual fitness in achieving its function.

Functional stability

For functional components to persist they must display some degree of stability in fitness to function.

Functional adaptation $\quad$ For functional components to persist in a changing environment they must have the ability to adapt their function. One of the most important domains of adaptation is cooperation. Functional components must interact according to the principles of decentralized cooperation in order to maximize outcomes for each. Functions must be prioritized according to the principles of centralized cooperation in order to maximize outcomes globally.

Table 2: Components of the General Collective Intelligence model.

\section{Cooperation as an Adaptive Domain}

As mentioned, living organisms can be represented in terms of a number of adaptive domains. Collections of living organisms might also be represented as having virtual adaptive domains. One of these adaptive domains is cooperation. In the same way that multicellular organisms must obey certain rules of cooperation in order for cooperation between cells to be stable, in order to scale any collective reasoning over a great many participants those participants must obey the same set of rules governing stable cooperation.

\begin{tabular}{|l|l|}
\hline Adaptive Processes (Basic Life Processes) \\
\hline Life Process & Name \\
\hline L1 & Homeostasis \\
\hline L2 & Autopoiesis (Reproduction) \\
\hline L3 & Growth (Maximize Outcomes) \\
\hline L4 & Evolve \\
\hline L5 & $\begin{array}{l}\text { Act (implemented by the "collective body" at } \\
\text { the level of the physical awareness process that } \\
\text { chooses a path through sensory space, and }\end{array}$ \\
\hline
\end{tabular}




\begin{tabular}{|l|l|}
\hline implemented by the "collective consciousness" \\
\hline L6 & $\begin{array}{l}\text { ime } \\
\text { at the level of the conscious self-awareness } \\
\text { process that chooses a path through awareness } \\
\text { space.) }\end{array}$ \\
\hline L7 & Cooperation (between functional units) \\
\hline L8 & $\begin{array}{l}\text { Motivation (implemented by the "collective } \\
\text { emotions") }\end{array}$ \\
\hline
\end{tabular}

Table 3: Adaptive processes corresponding to domains of adaptation proposed by the Functional Modeling Framework.

In order to create the potential to scale cooperation to the point it creates sufficient value that participation in that cooperation is self-sustaining, cooperation must have the potential to optimize some collective outcome as opposed to optimizing outcomes for some subset of participants. In order to always have the potential capacity to optimize some collective outcome for a group of entities then that optimization must be within the control of those entities. Or in other words, all processes by which such outcomes are achieved must consist of activities executed by those entities. As opposed those entities having their outcomes determined through processes they don't participate in.

In the domain of adaptation through cooperation, decentralized cooperation in which entities participate in achieving outcomes must be balanced with centralized decision-making in which entities are directed to achieve outcomes. Decentralization of decision-making in collective processes provides the capacity to maximize outcomes for all participants in the group equally, or in other words to give each participant an equal slice of the pie. Centralization of decision-making in collective processes provides the capacity to prioritize functions that are more important in maximizing outcomes that can be achieved by the group. Or in other words to increase the size of the pie. Centralization and decentralization must be balanced in order to optimize collective outcomes and ensure cooperation is stable.

While the ability to objectively optimize outcomes is important, no system of optimization, regardless how good, can work if it isn't used. Therefore a system of decision-making which ensures its own use is key to achieving this optimization. All these requirements place strict constraints on the way every element of a GCI must be designed. These constraints are expressed through a set of principles of intelligent cooperation which ensure that all available functionality, all participants, and other elements of a GCI are actually used, and are used in their optimal context. By ensuring that all processes engaged in by the group are required to adhere to the principles of intelligent cooperation, then if GCI has the capacity to reliably achieve this optimization, optimization of collective outcomes might be reliably achieved. 


Proposed Principles of Intelligent Cooperation
\begin{tabular}{|l|l|} 
Force Towards & Criteria \\
\hline Centralization & $\begin{array}{l}\text { Prioritize selection of processes to executed according to fitness } \\
\text { in achieving collective outcomes so that it is possible to } \\
\text { maximize those outcomes. }\end{array}$ \\
\hline Decentralization & $\begin{array}{l}\text { Operation of processes is Peer-to-Peer (no third party can insert } \\
\text { their interests) }\end{array}$ \\
\hline Decentralization & $\begin{array}{l}\text { Operation of processes is Decentralized (process activities are } \\
\text { assigned to roles rather than specific entities) }\end{array}$ \\
\hline Decentralization & $\begin{array}{l}\text { Operation of processes is User-Centric (no third party } \\
\text { information is required) }\end{array}$ \\
\hline Decentralization & $\begin{array}{l}\text { Operation of processes is Massively Collaborative (no pre- } \\
\text { defined limits to scaling participation) }\end{array}$ \\
\hline Decentralization & $\begin{array}{l}\text { Determine the relative value of work in a universal way that } \\
\text { enables any entity to participate in collective processes } \\
\text { (collective reasoning processes in the case of GCI). }\end{array}$ \\
\hline Decentralization & $\begin{array}{l}\text { Determine the relative value of resources to enable more open } \\
\text { sharing of those resources so executing collective processes } \\
\text { based on them is possible. }\end{array}$ \\
\hline Decentralization & $\begin{array}{l}\text { Define a metric of fitness for each participant to open } \\
\text { participation to better participants that might replace it. }\end{array}$ \\
\hline Decentralization & $\begin{array}{l}\text { For each process define a metric of fitness enabling selection of } \\
\text { the best process to participate in. }\end{array}$ \\
\hline
\end{tabular}

Table 4: Principles in the domain of adaptation through cooperation.

Since the domain of cooperation is represented as having its own functional state space, these principles might also potentially be expressed as a set of basic functions through which systems navigate that cooperation domain.

The implications of this cooperation domain are critical. Participants in processes can be individuals in the case of collective cognition or functional components of cognition in the case of individual cognition. In either case, centralization constrains system so that they cannot solve problems in which solutions are not aligned with the interests of the participants in which control over decision-making is centralized. In addition to making groups unable to discover these classes of solutions, centralization also makes groups unable to define problems in certain ways. Functionality must be decentralized across all participants to define problems in a way that maximizes impact on the problem as perceived by the entire group, rather than the definition of the problem becoming aligned with the interests of some subset of participants. To achieve this decentralization in adaptation itself, nature must take a modular approach that separates adaptive processes into different domains and that chooses which adaptive functionality to put in each. This choice must be made according to the principles of intelligent cooperation between adaptive processes if the set of adaptive domains is to have the capacity to maximize adaptive fitness across all domains. In other words, rather than defining a GCI as a single adaptive system, adaptive domains in a GCI must be limited in their functionality (modular and reuseable) so they can be adapted without having to change the entire system. As a result some of the 
constraints against problem definition and problem solving might exist in each adaptive domain. For example, each adaptive domain might lack the capacity to change its own adaptive functions. Therefore each adaptive domain must exist in a hierarchy of other adaptive domains if the constraints to its adaptability are to be removable. In summary, the various adaptive domains might be required to achieve true general problem-solving ability.

These principles together with general problem-solving ability lead to the uniqueness of GCI, which is expected to be the capacity to execute self-organizing processes that might enable groups to selfassemble into potentially massive networks of cooperation that can target any impact, that can radically increase the probability of achieving that impact, that can radically increase the magnitude of that impact, and that can create sufficient value to make that impact self-sustaining. Nature targets outcomes through self-assembling processes that grow, evolve, or become more fit in other adaptive domains, so life can find the resources to feed its own increase in ability to target those outcomes from resources that are already there in the environment. All the adaptive processes that nature spent billions of years researching and perfecting might potentially be modeled and replicated within a GCI. For example, rather than nonprofits competing for donor funding, a GCI might organize cooperation between thousands of NGOs to radically increase social, economic, or other impacts per dollar of program funds so programs are self-funding once launched. Rather than developing new networks of cooperation, in its "genome" a GCI might store optimized networks of cooperation processes between potentially billions or more individuals or organizations, and use an evolution algorithm, or use a reproduction or other adaptive algorithm, to adapt an existing network. A conceptual case study of a network of only five organizations suggests that increasing social impact per dollar by a thousand fold might be reliably achievable [25]. And that rather than being limited to the social impact possible with any finite amount of funding, social impact might be made sustainably self-funding so impact might be achieved at the scale required to transform communities globally. If so, then poverty, and other human suffering might already have reliably achievable solutions.

\section{Exponentially Increasing General Problem-Solving Ability}

As opposed to all current collective intelligence methodologies or software platforms [21]-[25], GCI has a number of properties that might exponentially increase the general problem-solving ability of the group over that of any individual. GCI firstly has the potential to combine individual cognition into a single virtual collective cognition with general problem-solving ability. Secondly, GCI defines what is believed to be the first complete semantic representation to model collective reasoning processes and information so that it's possible to exchange understanding at exponentially greater speed, rather than just exchanging information. Thirdly, GCI creates the possibility of a "big bang" in the collective conceptual space in which the size and density of that space increase exponentially, in addition to increasing the rate at which that conceptual space might be navigated.

An exponential increase in the size and density of the collective conceptual space can potentially occur in a GCI because any reasoning is itself represented by a concept in conceptual space. Therefore any new reasoning path between two concepts is an interaction that creates a new point in conceptual space. A problem in conceptual space is represented by the lack of a path between an initial concept and a final concept. A generalization in conceptual space is represented by a concept occupying a larger region in conceptual space that contains smaller regions representing more well-defined concepts. As the generalization becomes larger, the number of reasoning paths between that generalization and concepts within that generalization increases in proportion to the concepts contained within the generalization. As the generalization increases in size to the point that it includes both the initial concept defining a given problem as well as the final concept, the number of reasoning paths rises to the square of the number of concepts. This can be intuitively understood as all as saying that concepts 
can potentially interact when both regions overlap. Since each interaction creates a concept, the potential interactions might lead to an exponential increase in the number of concepts. At this point the size and density of the conceptual space can increase exponentially. By exponentially increasing the number of entities involved in the cognition process, and by executing processes in parallel, the rate at which the conceptual space can be navigated might also be increased.

Since intelligence from the functional modeling perspective is believed to be the volume of conceptual space that can be navigated per unit of time, exponentially increasing the size and density of conceptual space and exponentially increasing the rate at which that space can be navigated might potentially result in an exponential increase in collective intelligence. The meaning of an exponential increase in collective intelligence in practice is the capacity to define self-organizing processes that can engage massive networks in solving any problem, that can generalize in order to reuse solutions from other domains wherever they apply, and that can reliably navigate far more broad and more complex problems.

Having general problem-solving ability, a GCI must have the ability to execute any collective intelligence algorithms, as well as AI, bio-inspired, or other computing solutions. Such solutions might be added to a library of reasoning processes that a GCI might use to increase its general problem solving ability. Without the ability for semantic representation, and therefore without the ability to differentiate and search for the functionality in each collective reasoning process, there may not be the ability to use that library intelligently. Any given collective cognitive process might be analogous to either one of the two possible human reasoning processes, rational methodical (logical) reasoning, or intuitive reasoning. Intuitive reasoning is a black box in that subjects might not be able to explain it. While collective intelligence solutions based on estimation from past patterns might model intuition, and therefore always remain black boxes, collective intelligence based on rational methodical reasoning must expose their logic if that logic is to be analyzed and compared. Without representing the logic in any logical reasoning based procedures in terms of universally comparable collective cognitive functions, any logical process in such a library remains a black box that can't be distinguished by its internal workings.

Implementing all of the components of GCI, and gaining the capacity to semantically represent any given collective reasoning process in its entirety might be necessary to achieve complete general problem solving ability. However, preliminary results from case studies analyzing the potential impact of software platform designs based on this GCI model [25] suggest that implementing only part of the model has the potential to significantly optimize decision-making by itself.

\section{Validation of the Model and Proposed Implementation in Software Platforms}

General problem solving ability requires the ability to target any outcome. Increasing this problem solving ability implies an increase in ability to achieve outcomes. Using impact on the volume of collective outcomes as a measure of collective problem solving ability, ways to increase this impact are increasing the probability of achieving targeted outcomes, increasing the magnitude of targeted outcomes, and increasing the duration of the outcome by making it self-sustaining. The General Collective Intelligence (or "Change Engine") approach was used to design an Agricultural Livelihoods Program as phase I of a proposed ten phase Collective Intelligence based Program to Accelerate Achievement of the Sustainable Development Goals (CIPAA-SDGs) targeting a wide variety of SDGs. The design of the program validated the ability of this approach to target a range of outcomes, and projections for the approach validated its potential to increase the probability of achieving targeted outcomes, to multiply outcomes (by up to 750 times), and to become self-sustaining [26]. 
In this Agricultural Livelihoods program a number of agricultural value chains have been proposed. These value chains define collectively intelligence processes of cooperation to achieve increases in collective impact. Ongoing work is exploring the suggestion that by defining these processes, each value chain can potentially be added to a library of functions the GCI can use to achieve collective impact.

Current work is exploring the claim that wherever the value of cooperation is greater than zero, a GCI platform can potentially use algorithms to remove the barriers to finding these opportunities to cooperate, so that cooperation can be increased to the point that its value acts as a subsidy that can potentially increase the probability of achieving impact on collective challenges significantly, creating the potential to greatly increase the magnitude of that impact, and creating the potential to make that impact self-sustaining. While the conceptual case study of the Social Impact Marketplace platform design created using this approach suggested that GCI has the potential to automate processes of collectively intelligent cooperation to achieve an up to $750 \mathrm{X}$ increase in impact per program dollar when applied to improve agricultural livelihoods, another conceptual case study of a platform design created using this approach is exploring the suggestion that GCI has the potential for sufficient cost savings in healthcare to potentially pay for universal health coverage for those without.

In general, GCI has profound implications in any products or services that involve group processes in any part of their business life-cycle (from research and development to recycling), or that are used in group processes. This includes virtually every product or service. With GCI, significant improvements in the collective impact of those products and services are proposed to be reliably achievable. In fact, as mentioned, game theory modeling is currently being used to explore the suggestion that in the group decision-making processes governing social impact and sustainable development, centralization and alignment of groups with those centralized interests might create perverse incentives that prevent solution of such collective challenges from being reliably achievable at all without GCI. If so, these perverse incentives suggest that without GCI, collective impact that requires such solutions, including sustainable economic development or "green growth", might not be reliably achievable. However current work is also exploring the suggestion that with GCI, collective impact might reliably be achieved.

Without a GCI group outcomes might be predominantly aligned with interests of individual group members, and individuals who don't primarily compete to further their own interests rather than contributing to the collective well-being gradually might lose access to resources and therefore lose decision-making power. As a result outcomes might be dominated by individuals who compete to increase consumption, inequality, and other harm to collective well-being that enables them to win this competition. This alignment has been suggested to be a systemic force towards individual outcomes that prevents collective impact such as sustainable green growth, amelioration of environmental degradation associated with climate change, or achievement of the other Sustainable Development Goals (SDGs), from being reliably attainable [27]. With GCI, the situation is proposed to be completely reversed, with outcomes being predominantly aligned with collective interests, so it is the individuals who don't cooperate to further collective interests who gradually lose decision-making power, providing competitive advantage to cooperating groups so that collective outcomes like the SDGs become reliably achievable.

\section{Conclusions}

GCI is potentially a paradigm shift in group organization. Since an entity with a well-defined cognition with it's own independent thread of reasoning must behave as an independent actor, GCI is a radically different concept from Collective Intelligence (CI) solutions, which are essentially collective decision- 
making tools controlled by some subset of actors within or outside the group. Where Collective Intelligence tools potentially cannot be reliably separated from being aligned with the interests of their designers, owners, and other decision-makers, being driven by the collective cognition a General Collective Intelligence platform must reliably be aligned with the collective interests of the group.

\section{References}

[1] Woolley, Anita Williams; Chabris, Christopher F.; Pentland, Alex; Hashmi, Nada; Malone, Thomas W. (29 October 2010). "Evidence for a Collective Intelligence Factor in the Performance of Human Groups". Science. 330 (6004): 686-688. Bibcode:2010Sci...330..686W. doi:10.1126/science.1193147. PMID 20929725. S2CID 74579.

[2] Krafft, Peter M. "A simple computational theory of general collective intelligence." Topics in cognitive science 11.2 (2019): 374-392.

[3] Wolf, Max, et al. "Collective intelligence meets medical decision-making: the collective outperforms the best radiologist." PloS one 10.8 (2015): e0134269.

[4] Andres, Frederic, et al. "Collective intelligence management on digital agriculture resources."

Software, Knowledge, Information Management and Applications (2008): 112-117.

[5] Nick Bostrom, Get ready for the dawn of superintelligence, New Scientist, Volume 223, Issue 2976, 2014, Pages 26-27, ISSN 0262-4079, https://doi.org/10.1016/S0262-4079(14)61304-X

[6] Malone, Thomas W. Superminds: The surprising power of people and computers thinking together. Little, Brown Spark, 2018.

[7] Heylighen, Francis, and Marta Lenartowicz. "The Global Brain as a model of the future information society: An introduction to the special issue." (2017): 1-6.

[8] Last, Cadell. "Global brain: foundations of a distributed singularity." The 21st Century Singularity and Global Futures. Springer, Cham, 2020. 363-375.

[9] Williams, Andy E. "A Model for Human, Artificial \& Collective Consciousness (Part I)." Journal of Consciousness Exploration \& Research 10.4 (2019).

[10] Williams, Andy E. "A Model for Human, Artificial \& Collective Consciousness (Part II)." Journal of Consciousness Exploration \& Research 10.4 (2019).

[11] Williams A.E. (2020) A Model for Artificial General Intelligence. In: Goertzel B., Panov A., Potapov A., Yampolskiy R. (eds) Artificial General Intelligence. AGI 2020. Lecture Notes in Computer Science, vol 12177. Springer, Cham. https://doi.org/10.1007/978-3-030-52152-3 38

[12] B. CHANDRASEKARAN, Representing function: Relating functional representation and functional modeling research streams, Artificial Intelligence for Engineering Design, Analysis and Manufacturing 2005, 19, 65-74, DOI: 10.10170S0890060405050079

[13] Zhang, W.Y., Tor, S.Y. \& Britton, G.A. , Managing modularity in product family design with functional modeling, The International Journal of Advanced Manufacturing Technology(2006) 30: 579. https://doi.org/10.1007/s00170-005-0112-z

[14] Eisenbart, B., Gericke, K., \& Blessing, L. (2013). An analysis of functional modeling approaches across disciplines. Artificial Intelligence for Engineering Design, Analysis and Manufacturing, 27(3), 281-289. doi:10.1017/S0890060413000280

[15] Zhengrong Cheng, Yongsheng Ma, A functional feature modeling method, Advanced Engineering Informatics, Volume 33, 2017, Pages 1-15, ISSN 1474-0346, https://doi.org/10.1016/j.aei.2017.04.003. [16] Tensa, M., Edmonds, K., Ferrero, V., Mikes, A., Soria Zurita, N., Stone, R., \& DuPont, B. (2019). Toward Automated Functional Modeling: An Association Rules Approach for Mining the Relationship between Product Components and Function. Proceedings of the Design Society: International Conference on Engineering Design, 1(1), 1713-1722. doi:10.1017/dsi.2019.177

[17] Sten Bay Jørgensen, Morten Lind, and Niels Jensen, Functional Modeling View on Product and Process Engineering in Design and Operations

Industrial \& Engineering Chemistry Research 201958 (26), 11129-11148 
DOI: $10.1021 /$ acs.iecr.8b06338

[18] Zhao, Meng, et al. "A State-Behavior-Function Model for Functional Modeling of Multi-State Systems." Proceedings of the Institution of Mechanical Engineers, Part C: Journal of Mechanical Engineering Science, vol. 233, no. 7, Apr. 2019, pp. 2302-2317, doi:10.1177/0954406218791640. [19] Kahneman, D. (2012).Thinking, fast and slow.London: Penguin Books. Isbn: 0141033576. [20] Williams, A. E. (n.d.). Deducing the Properties Required by General Collective Intelligence Platforms. Retrieved from osf.io/preprints/africarxiv/243wx

[21] J. Salminen, Collective intelligence in humans: A literature review, CoRR abs/1204.3401 (2012).

[22] Shweta Suran, Vishwajeet Pattanaik, and Dirk Draheim. 2020. Frameworks for Collective Intelligence: A Systematic Literature Review. ACM Comput. Surv. 53, 1, Article 14 (February 2020), 36 pages. DOI:https://doi.org/10.1145/3368986

[23] Solberg Søilen, Klaus, Making sense of the collective intelligence field: A review, 2019 (English), Journal of Intelligence Studies in Business, ISSN 2001-015X, E-ISSN 2001-015X, Vol. 9, no 2, p. 6-18 [24] Yu, C., Chai, Y. and Liu, Y. (2018), "Literature review on collective intelligence: a crowd science perspective", International Journal of Crowd Science, Vol. 2 No. 1, pp. 64-73. [25] Javier Del Ser, Eneko Osaba, Daniel Molina, Xin-She Yang, Sancho Salcedo-Sanz, David Camacho, Swagatam Das, Ponnuthurai N. Suganthan, Carlos A. Coello Coello, Francisco Herrera, Bioinspired computation: Where we stand and what's next, Swarm and Evolutionary Computation, Volume 48, 2019, Pages 220-250, ISSN 2210-6502, https://doi.org/10.1016/j.swevo.2019.04.008. [26] Williams, A. E. (2020, April 30). The Collective Intelligence based Program to Accelerate Achievement of the Sustainable Development Goals as a Case Study for Collectively Intelligent Program Design. https://doi.org/10.31235/osf.io/r2dxq [27] Andy E Williams. The Need for General Collective Intelligence in Addressing the Sustainable Development Goals, 19 March 2020, PREPRINT (Version 1) available at Research Square [https://doi.org/10.21203/rs.3.rs-18022/v1] 